\title{
Wavelength-Diverse Polarization Modulators for Stokes Polarimetry
}

\author{
Steven Tomczyk, Roberto Casini, Alfred G. de Wijn and Peter G. Nelson \\ High Altitude Observatory, National Center for Atmospheric Research, \\ P. O. Box 3000, Boulder, CO 80307-3000, U.S.A.
}

\begin{abstract}
Information about the three-dimensional structure of solar magnetic fields is encoded in the polarized spectra of solar radiation by a host of physical processes. To extract this information, solar spectra must be obtained in a variety of magnetically sensitive spectral lines at high spatial, spectral, and temporal resolution with high precision. The need to observe many different spectral lines drives the development of Stokes polarimeters with a high degree of wavelength diversity. We present a new paradigm for the design of polarization modulators that operate over a wide wavelength range with near optimal polarimetric efficiency and are directly applicable to the next generation of multi-line Stokes polarimeters. These modulators are not achromatic in the usual sense because their polarimetric properties vary with wavelength, but they do so in an optimal way. Thus we refer to these modulators as polychromatic. We present here the theory behind polychromatic modulators, illustrate the concept with design examples, and present the performance properties of a prototype polychromatic modulator. (c) 2018 Optical Society of America
\end{abstract}

\section{Introduction}

Our knowledge of solar magnetism relies heavily on our ability to detect and interpret the polarization signatures of magnetic fields in solar spectral lines. Since the identification of sunspots as regions of strong magnetism by means of the observation of the Zeeman effect [1], the number of physical mechanisms applied to the interpretation of solar spectral line profiles has increased significantly. The Hanle effect observed in linear polarization has been exploited to diagnose weak turbulent magnetic fields [2 4]. A rich spectrum of linear polarization observed near the solar limb named the "second solar spectrum" [5] has been used to constrain magnetic fields and scattering physics. Observations of lines like Mn which display hyperfine splitting have been used to break the degeneracy between magnetic flux and field for weak magnetic fields [6 [6]. Information about plasma kinetics during solar flares is encoded in the linear polarization of $\mathrm{H}$ lines through impact polarization [9].

Other important justifications for multi-line observations exist. Foremost among these is that observations of spectral lines formed at different heights in the solar atmosphere are needed to 
constrain the magnetic field geometry in three dimensions. Secondly, simultaneous observation of multiple spectral lines and the application of a line-ratio technique have been shown to greatly enhance the diagnostic potential of Zeeman-effect observations [10]. Finally, improving technologies (e.g. IR detector arrays) have made it possible to take advantage of the increased sensitivity of the Zeeman effect with wavelength through the observation of infrared spectral lines [11,12].

It follows that the next generation of Stokes polarimeters must have the capability to observe the solar atmosphere in a variety of spectral lines over a wide wavelength range, coupled with the ability to observe several lines simultaneously. This is reflected in the design of the recently completed Spectro-Polarimeter for InfraRed and Optical Regions (SPINOR, 13]), installed at the Dunn Solar Telescope (DST) of the National Solar Observatory on Sacramento Peak (NSO/SP, Sunspot, NM), that can observe between 430 and $1600 \mathrm{~nm}$. Post-focus instruments at the recently completed New Solar Telescope will observe between 400 and $1700 \mathrm{~nm}$ [14. The suite of spectropolarimeters planned for the 4-m Advanced Technology Solar Telescope will cover the wavelength range between 380 and $2500 \mathrm{~nm}$ [15]. And the proposed science for the planned 4-m European Solar Telescope [16] emphasizes multi-wavelength observations from the UV to the near-IR.

One immediate instrument requirement stemming from this need for wavelength diversity is that the polarization modulation scheme must be efficient at all wavelengths of interest. Typically, one attempts to achieve this goal by achromatizing the polarimetric response of a modulator. This, for instance, is the rational behind the design of super-achromatic waveplates [17, 18]. In this paper, instead, we present a new paradigm for the design of efficient polarization modulators that are not achromatic in the above sense, since they have polarimetric properties that vary with wavelength. However, they do so in such a way that they can be operated over a wide wavelength range with near optimal polarimetric efficiency. We refer to these modulators as polychromatic. Because of their performance, these are directly applicable to the next generation of multi-line Stokes polarimeters. In the next section, we summarize the basic theory behind polarization modulators. Next, we present example designs using existing technologies, including a discussion of the method used to obtain them. Finally, the performance properties of a prototype polychromatic modulator are presented.

\section{Theory}

Most polarimeters operate by employing retarders and polarizers in a configuration that encodes polarization information into a modulated intensity signal. This intensity modulation is measured with a detector and analyzed to infer the input polarization state. An extensive treatment of the theoretical operation of Stokes polarimeters has been given in [19,20]. This work draws extensively from that formalism.

The complete polarization state of an input light beam can be described by the Stokes vector:

$$
\boldsymbol{S} \equiv(I, Q, U, V)^{T}
$$

where $I$ is the intensity, $Q$ and $U$ are the net linear polarizations measured in two coordinate frames that are rotated by $45^{\circ}$ with respect to each other, and $V$ is the net circular polarization. Since

the Stokes vector contains four unknown parameters, any polarimeter must make a minimum of 
four measurements to determine it. The polarization properties of a polarimeter in any state can be conveniently described by a $4 \times 4$ Mueller matrix in the usual way. The first row of any Mueller matrix captures the transformation of a Stokes vector into intensity. Assuming that the detector is sensitive to intensity alone, then only the first row of the polarimeter Mueller matrix is important.

The modulation of intensity by a $n$-state polarimeter is captured in the $n \times 4$ modulation matrix, M. For each state, the corresponding row of the modulation matrix (the modulation vector) is given by the first row of the Mueller matrix of the polarimeter in that state. The modulation matrix is generally normalized by the $(1,1)$ element. Note that, for a retarder-based modulator, the first element of the modulation vector is the same in all modulation states, so after normalization the first column of the modulation matrix is all made of $1 \mathrm{~s}$. The operation of a polarimeter can then be represented by:

$$
\boldsymbol{I}=\mathbf{M} \boldsymbol{S}
$$

and the input Stokes vector is obtained by

$$
S=\mathrm{D} I
$$

where $\mathbf{D}$ is the demodulation matrix. In 20$]$ it is shown that, for a given modulation matrix, $\mathbf{M}$, the optimal demodulation matrix is $\mathbf{D}=\left(\mathbf{M}^{T} \mathbf{M}\right)^{-1} \mathbf{M}^{T}$. The polarimetric efficiency of a modulation scheme can be derived from the demodulation matrix itself, and is given by [19]:

$$
\epsilon_{i}=\left(n \sum_{j=1}^{n} D_{i j}^{2}\right)^{-1 / 2}
$$

where the subscript $i$ varies over the four elements of the Stokes vector. The polarimetric efficiency is important in that it quantifies the noise propagation through the demodulation process. The noise in the $i$-th element of the inferred Stokes vector, $\sigma_{i}$, depends directly on the efficiency as [20]:

$$
\sigma_{i}=\frac{1}{\sqrt{n}} \frac{\sigma_{I}}{\epsilon_{i}}
$$

where $\sigma_{I}$ is the uncertainty on the measured intensity, assumed constant, during the measurement cycle. Equation 5 implies that an efficient polarization modulator is required to measure a Stokes vector with high precision. The efficiency on any Stokes parameter can be between 0 and 1 , subject to the two independent constraints $\epsilon_{Q}^{2}+\epsilon_{U}^{2}+\epsilon_{U}^{2} \leq 1$, and $\epsilon_{I}^{2} \leq 1$. Many so-called magnetographs are designed to modulate only Stokes $V$ with high efficiency. In this paper, we are concerned with modulation schemes that are balanced, in the sense that the efficiencies of Stokes $Q, U$, and $V$ are equal. In this case, the maximum (i.e., optimal) modulation efficiency for Stokes $I$ is 1 , and for Stokes $Q, U$, and $V$, is $1 / \sqrt{3} \approx 57.7 \%$.

There are several configurations for optimally efficient polarization modulators at a single wavelength. These may require different numbers of modulation states to achieve the full measurement of the Stokes vector. In Table 1, we present examples of optimally efficient and balanced modulators at a single wavelength, employing three different modulator technologies. These are a rotating 


\begin{tabular}{|c|c|c|c|}
\hline Modulator Type & Retardance & Orientation & Modulation Vector \\
\hline \multirow[t]{4}{*}{ Stepped Retarder } & $131.8^{\circ}$ & $-51.7^{\circ}$ & $(1,-1 / \sqrt{3},+0.375,+0.725)$ \\
\hline & $"$ & $-15.1^{\circ}$ & $(1,+1 / \sqrt{3},-0.725,+0.375)$ \\
\hline & $"$ & $+15.1^{\circ}$ & $(1,+1 / \sqrt{3},+0.725,-0.375)$ \\
\hline & $"$ & $+51.7^{\circ}$ & $(1,-1 / \sqrt{3},-0.375,-0.725)$ \\
\hline \multirow[t]{6}{*}{ LCVRs $(\# 1, \# 2)$} & $\left(180^{\circ}, 360^{\circ}\right)$ & $\left(0^{\circ}, 45^{\circ}\right)$ & $(1,+1,0,0)$ \\
\hline & $\left(180^{\circ}, 180^{\circ}\right)$ & $"$ & $(1,-1,0,0)$ \\
\hline & $\left(90^{\circ}, 90^{\circ}\right)$ & $"$ & $(1,0,+1,0)$ \\
\hline & $\left(90^{\circ}, 270^{\circ}\right)$ & $"$ & $(1,0,-1,0)$ \\
\hline & $\left(180^{\circ}, 90^{\circ}\right)$ & $"$ & $(1,0,0,+1)$ \\
\hline & $\left(180^{\circ}, 270^{\circ}\right)$ & $"$ & $(1,0,0,-1)$ \\
\hline \multirow[t]{4}{*}{$\operatorname{FLCs}(\# 1, \# 2)$} & $\left(180^{\circ}, 102.2^{\circ}\right)$ & $\left(0^{\circ},-18.1^{\circ}\right)$ & $(1,+1 / \sqrt{3},+1 / \sqrt{3},-1 / \sqrt{3})$ \\
\hline & $"$ & $\left(0^{\circ},+18.1^{\circ}\right)$ & $(1,+1 / \sqrt{3},-1 / \sqrt{3},+1 / \sqrt{3})$ \\
\hline & $"$ & $\left(45^{\circ},+18.1^{\circ}\right)$ & $(1,-1 / \sqrt{3},+1 / \sqrt{3},+1 / \sqrt{3})$ \\
\hline & $"$ & $\left(45^{\circ},-18.1^{\circ}\right)$ & $(1,-1 / \sqrt{3},-1 / \sqrt{3},-1 / \sqrt{3})$ \\
\hline
\end{tabular}

Table 1. Examples of optimal modulation schemes for one wavelength, based on typical retarding devices. The retardances (second column) are given at the optimization wavelength. The orientation of the fast axes (third column) are positive counterclockwise, when looking at the light source. The modulation vector (last column) for a given $i$-th state corresponds to the $i$-th row of the modulation matrix. The last example is the modulator solution adopted for the NSO/DLSP.

waveplate of fixed retardation, a pair of liquid crystal variable retarders (LCVRs) with variable retardation and fixed orientation of the fast axis (e.g., [21]), and a pair of ferroelectric liquid crystals (FLCs) with fixed retardance but variable orientation of the fast axis. The particular solution presented here for the FLC modulator is implemented in the Diffraction-Limited Spectro-Polarimeter (DLSP, [22]), which is also installed at the NSO/SP DST. An example of a FLC modulator that is optimally efficient but with higher efficiency for circular polarization is given in [23]. The LCVR modulator presented here uses 6 modulation states, although it is possible to create a LCVR modulator that is balanced and optimally efficient at one wavelength requiring only 4 states. We also note that a single LCVR cannot modulate all Stokes parameters, regardless of the number of modulation states.

The retardance of a given device is wavelength dependent so these standard recipes generally work over a limited wavelength range. One could change the recipe to access other wavelengths but 

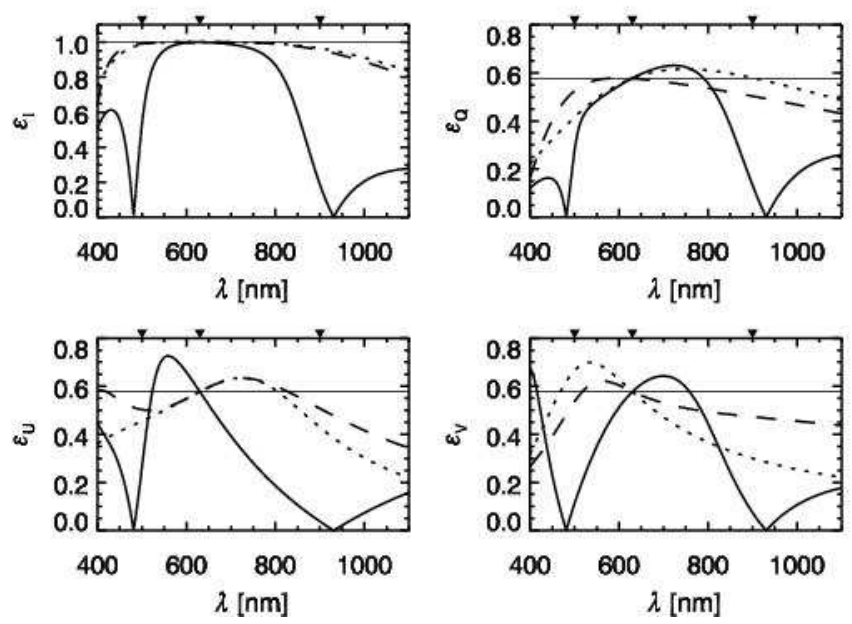

Fig. 1. Modulation efficiency curves for the four Stokes parameters between 400 and $1100 \mathrm{~nm}$ for modulators with two FLCs. Continuous curve: optimal and balanced solution at $630 \mathrm{~nm}$ (indicated by the central arrow) corresponding to the NSO/DLSP modulator [22]. Dotted curve: polychromatic solution corresponding to a simple modification of the NSO/DLSP modulator, where the first FLC is rotated by an additional angle of $67.5^{\circ}$. Dashed curve: polychromatic solution obtained from the former solution through the addition of a fixed quartz retarder between the two FLCs. This solution was optimized between 500 and $900 \mathrm{~nm}$ (indicated by the two outmost arrows). The horizontal solid lines in the four panels indicate the maximum theoretical modulation efficiencies that can be achieved simultaneously for the four Stokes parameters ( 1 for $I$, and $1 / \sqrt{3}$ for $Q, U$, and $V$ ).

simultaneous spectro-polarimetric observations in different spectral ranges would not be possible. The chromatic nature of the example FLC modulator is illustrated in Fig. 1 showing the variation of the efficiency with wavelength. In the plot, we assume a target wavelength of $630 \mathrm{~nm}$ and that the intrinsic birefringence of the FLCs is not a function of wavelength (i.e., no dispersion of the birefringence). We define the region of acceptable performance of a polarimeter as the region over which the efficiency is greater than the optimal efficiency divided by $\sqrt{2}$, that is, $1 / \sqrt{6} \approx 40.8 \%$. At this point, the increase in noise is equivalent to a reduction in the photon flux by a factor of 2, assuming Poisson statistics. By this definition, we find that the stepped-retarder solution is efficient over a spectral region of $268 \mathrm{~nm}$, the LCVR solution over $287 \mathrm{~nm}$, and the FLC solution over $128 \mathrm{~nm}$.

\section{Polychromatic Modulators with Near-Optimal Efficiency}

To create polarimeters that operate over a large wavelength range, instrument developers have typically tried to select optical materials in order to make the polarimeter achromatic; that is, to 
make the polarimeter modulation matrix as independent of wavelength as possible. The success of such an approach depends on the choice of materials and on the desired wavelength coverage. In principle, this affords an important simplification, which is the possibility of applying a single demodulation scheme to infer the Stokes vector at any wavelength. In practice, the application of a single demodulation scheme can only give a zeroth-order approximation to the true Stokes vector, because of the unavoidable residual wavelength dependence of the Mueller matrix of the modulator across the spectral range of interest. For example, the achromatic modulator of NSO/SPINOR shows a $15 \%$ variation of its retardance properties over its spectral range of operation (430-1600 $\mathrm{nm}, 24$ ). Therefore one still needs to perform a careful wavelength calibration of the polarimeter in order to infer the true Stokes vector within the requirements of polarimetric sensitivity dictated by the science. Previous work [25, 26] has also attempted to achromatize modulators by adding combinations of fixed and variable retarders, also with the goal of minimizing the wavelength dependence of the resulting Mueller matrix.

However, the preservation of a given form of the polarimeter's Mueller matrix with wavelength is a very limiting, and arguably unnecessary, constraint. The fundamental driver in the design of a polarization modulator for multi-line applications is the achievement of near-optimal modulation efficiencies in all Stokes parameters at all wavelengths of interest. The Mueller matrix of such a modulator can be completely arbitrary, and even strongly dependent on wavelength. Since calibration with wavelength is required, such wavelength dependence will not impact the precision of the polarimetric measurements. In addition, since the demodulation matrix can be calculated theoretically from the modulator design, it is straightforward to provide real-time approximations of the measured Stokes vector at all wavelengths of operation.

In designing the polarimeter for the 1-m Yunnan Solar Telescope (Yunnan Astronomical Observatory, China) [27] optimized the efficiency of their polarimeter over a broad wavelength range by adjusting the orientation of the fast axes of the retarding elements, and in our opinion created the first polychromatic modulator. However, [27] did not fully optimize their design by allowing the retardations of the elements to vary, they did not adopt an optimal demodulation scheme as described in [20] which could result in lowering the efficiency that is achieved, and they stressed the importance of minimizing crosstalk which is not necessary to achieve an optimally efficient polarimeter.

We propose a new paradigm where full-Stokes polarization modulators are designed to satisfy the constraint of having optimal and balanced polarimetric efficiency at all wavelengths of interest. This generally results in polarimeters that have modulation and demodulation matrices that are strong functions of wavelength. We refer to these modulators as polychromatic. We have developed a technique for optimizing polarization modulators using combinations of fixed and variable retarders. We find that varying the retardance and orientation of the optical components that comprise a modulator, and maximizing the polarimetric efficiency for all wavelengths of interest, results in realizable configurations with a high degree of wavelength diversity.

We employ an optimization code that systematically searches the parameter space for a solution that maximizes the Stokes modulation efficiencies over the desired spectral range. The search is 
performed following the strategy of Latin Hypercube Sampling [28] of the parameter space. This significantly improves the convergence speed of the search for the optimal solution, compared to a direct Monte Carlo sampling. Although the solutions found through this method are highly optimized, they may not be unique. Thus it is not possible to conclude with certainty that they represent the solutions with the highest possible efficiency for a given type of modulator. Different optimization schemes based on gradient methods have also been used with success (e.g., Powell, Levenberg-Marquardt), although they need realistic first guesses in order to converge properly.

We illustrate this concept with three different types of polychromatic modulators for polarimetric applications. For the first type of modulator, we consider a stack of two FLCs, with the possible addition of a fixed retarder to broaden the wavelength range. For this type of modulator, there are four modulation states. The broadening of the wavelength range of a FLC modulator by the addition of fixed retarders has been suggested [25] with the intention to minimize off-diagonal elements of the Mueller matrix, although that study did not result in efficient modulators over a wide wavelength range.

As a first example, we start with the FLC modulator used by the NSO/DLSP and presented in Table 1. The DLSP was designed to operate at $630 \mathrm{~nm}$ only. Figure 1 (continuous curve) shows that the DLSP, as configured, has optimal and balanced efficiency at $630 \mathrm{~nm}$ with a spectral range of acceptable performance of $128 \mathrm{~nm}$. We then optimized the efficiency of the DLSP modulator between 500 and $900 \mathrm{~nm}$, by allowing the orientation angles of the FLCs to vary. The resulting solution (dotted curve) is a simple modification of the NSO/DLSP configuration (cf. Table 1), where the first FLC is rotated such that the new switching angles are $67.5^{\circ}$ and $112.5^{\circ}$. With this new configuration, the modulator still provides optimal and balanced efficiency at $630 \mathrm{~nm}$, however the usable range is now twice as large as before $(259 \mathrm{~nm})$. Next, we added a fixed retarder between the two FLCs, and allowed the orientations of the FLCs and the retardance and orientation of the fixed retarder to vary. The resulting solution (dashed curve) yields a polarimeter with optimal and balanced efficiency at $630 \mathrm{~nm}$, but the usable range now spans $553 \mathrm{~nm}$. It must be noted that one can obtain even larger usable ranges with this type of modulator, when the optimization is extended to all the retarding devices. A clear example of this (even if still subject to some external constraints) is the ProMag modulator illustrated in Sect. 4.

The second type of modulator is based on a stack of retarders glued in a fixed set of relative orientations, that is then rotated as a whole to the required set of positions to perform the measurement of the Stokes vector. We consider a stepping modulator, rather than a continuously rotating one. The adoption of a continuous integration scheme results in a small reduction of the modulation efficiency. We refer to [29] for a typical estimation of the reduction of modulation efficiency for continuously rotating modulators. For this type of rotating modulator, we consider the usual scheme with 8 measurements at positions $k \pi / 8$, with $k=0, \ldots, 7$. The example in Fig. 2 shows the modulation efficiency of a stack of three retarders.

The third type is based on a stack of LCVRs followed by a fixed retarder. The number of states of this kind of modulator is a free parameter. Figure 3 considers a modulator with 6 states. The parameter space for this kind of modulator has a much higher dimensionality than for designs based 

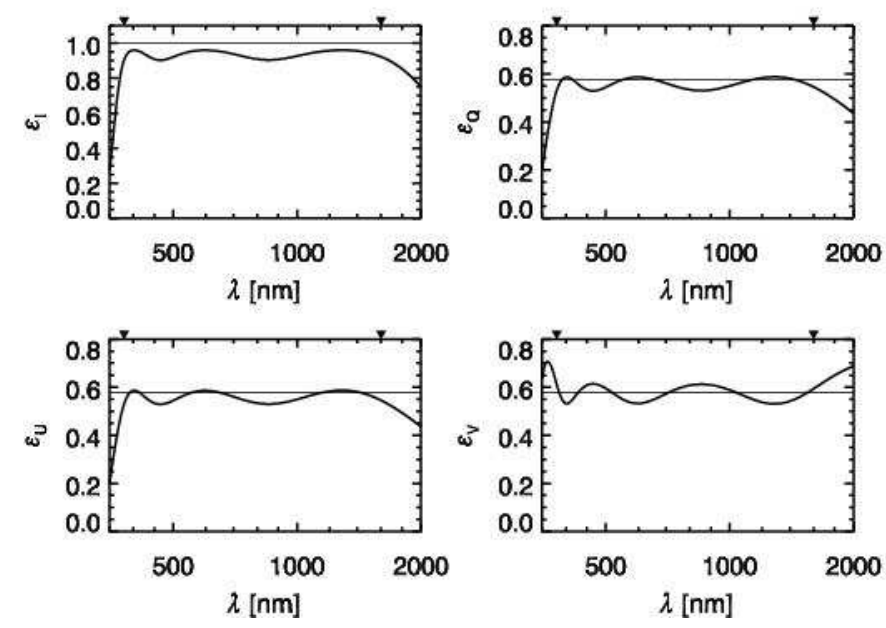

Fig. 2. As Fig. 1, but now for a modulator consisting of a stack of three quartz retarders rotated over 8 discrete steps of 22.5 degrees. The modulator was optimized between 380 and $1600 \mathrm{~nm}$ (indicated by the two arrows).

on FLCs or fixed retarders, and therefore multiple solutions with comparable efficiencies are often found. The example shown in Fig. 3 was determined by running the optimization several times and selecting the solution with the smallest deviation from optimal efficiency averaged over the optimization range.

In the solutions shown in Figs. 2 3 , the fast axis of the first device is conventionally fixed at $0^{\circ}$. We verified that this constraint does not affect the search of an optimally polychromatic solution for the modulator configuration. For practical purposes, we also assume that the fixed retarders are made of quartz, while for the LCVRs we assume no wavelength dispersion of the birefringence. While this limits the applicability of the recipes given in this paper, the implementation of realistic birefringence dispersion curves is possible. Preliminary tests show that this is a mildly limiting factor in the search of high-efficiency modulator configurations, typically resulting in a narrower spectral range of optimization for a given number of retarding devices.

Specific science applications of polarimeters may require giving a higher priority to selected spectral lines and/or Stokes polarization parameters. For this reason, in our optimization code both the optimizing wavelengths and the four Stokes parameters can be attributed different weights in the search of a high-efficiency modulator configuration. The solutions shown here were derived without any specific application in mind, and therefore they had equal weights for all optimizing wavelengths and Stokes parameters. In pratical situations, tweaking of some of the weights may be necessary to correct the behavior of the modulation efficiency in a particular interval of the spectral range. This is not surprising, especially in the optimization of the modulation efficiency over very large spectral ranges, since one needs to use a correspondingly large number of optimizing wavelengths (typically, of the order of 50), and the number of nearly equivalent solutions increases 

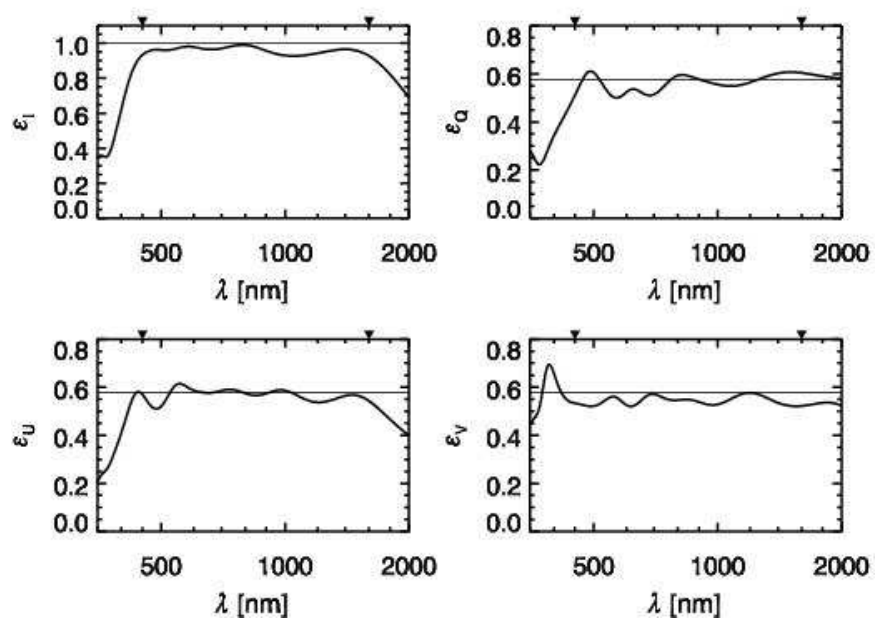

Fig. 3. As Fig. 2, but now for a modulator consisting of two LCVRs followed by a fixed quartz retarder, and using a 6-state modulation scheme. The modulator was optimized between 450 and $1600 \mathrm{~nm}$ (indicated by the two arrows).

dramatically because of the increased number of degrees of freedom in the optimization problem. In such cases, the use of different weights can help direct the search towards particular types of nearly equivalent solutions while excluding others, at the user's discretion.

\section{A Prototype Polychromatic Modulator}

As a first application of polychromatic modulators, we re-designed the polarimeter of the HAO Prominence Magnetometer (ProMag, [30]). This instrument was conceived to observe solar prominences and filaments in the spectral lines of $\mathrm{He} \mathrm{I}$ at 587.6 and $1083.0 \mathrm{~nm}$, and also in $\mathrm{H} \alpha(656.3 \mathrm{~nm})$. The re-design was prompted by a series of failed attempts at fabricating the ProMag modulator following the original design, which was based on a stack of six FLCs [30]. Thus, the original design was replaced by a much simpler scheme, utilizing only two FLCs plus a fixed retarder. The configuration was determined with the optimization method described above and constrained to use one of the FLCs from the original design. Retardances of the second FLC and the fixed quartz retarder were specified according to the optimized solution, and the retardances of these devices were measured in the laboratory. Since the measured retardances differed somewhat from the specified ones, a second optimization was performed varying only the orientations of the acquired devices using their measured retardances. The modulator was then constructed according to the design resulting from the second optimization. We estimate that the elements were oriented to the design values to within approximately $0.3^{\circ}$. Figure 4 shows the predicted efficiencies for the Stokes parameters resulting from the optimization. Actual efficiencies at 587.6 and $1083.0 \mathrm{~nm}$ were measured after deployment of the instrument at the Evans Solar Facility of the NSO/SP, and they are in good agreement with the theory (see crosses in Fig. (4). The discrepancy observed at $1083.0 \mathrm{~nm}$ is likely 

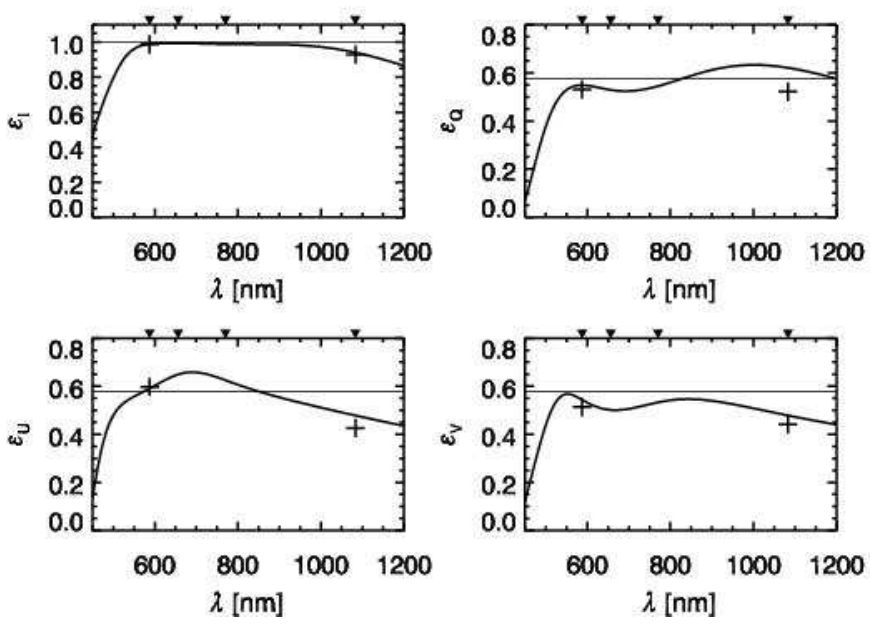

Fig. 4. Theoretical efficiency curves for the ProMag modulator, consisting of two FLCs followed by a quartz retarder. The modulator was optimized at 587.6, 656.3, 769.9 , and $1083.0 \mathrm{~nm}$ (indicated by arrows). The crosses indicate the measured efficiencies at 587.6 and $1083.0 \mathrm{~nm}$ after deployment of the instrument.

caused by a less precise determination of the retardances of the modulator optics at that wavelength, possibly due to an undetected leak in the interference filter used during the measurement in the laboratory, combined with the different spectral responses of the ProMag Alpha-NIR camera and the photo-diode used in the lab measurement.

The theoretical variation of the modulation matrix, M, with wavelength is shown in Fig. 5. The first column is unity and therefore is omitted. Measured modulation amplitudes at 587.6 and 1083.0 $\mathrm{nm}$ are also shown to be in close agreement with the theory.

\section{Conclusions}

In this paper we presented a new paradigm for the design of polarization modulators that responds to the needs of multi-line spectro-polarimetry. We argued that the effort of achromatizing the response matrix of a polarimeter - that is, trying to make the Mueller matrix as little dependent on wavelength as possible - is both a very limiting and unnecessary constraint. The only requirement that should be imposed on a polarimeter, in order to reach a given target of polarimetric sensitivity, is that its modulation efficiency be sufficiently high at all wavelengths of interest. As these wavelengths may be distributed over very large spectral ranges (of the order of $1000 \mathrm{~nm}$ or larger), and because of the wavelength dependence of the optical properties of retarding devices, the design of optimally efficient polarization modulators from first principles is a particularly arduous, if not impossible, task. For this reason, we have developed an improved Monte Carlo search technique in order to explore the parameter space of a polarimeter, and identify optimally efficient solutions compatible with the particular type of modulator. We illustrated the power of this method by im- 

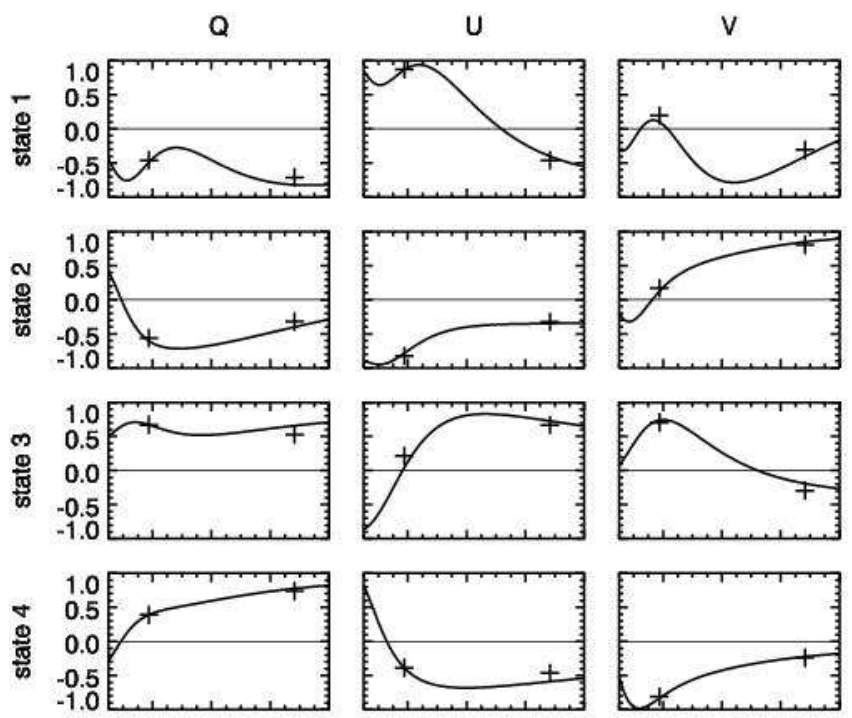

60080010001200

$\lambda[\mathrm{nm}]$

$\lambda[\mathrm{nm}]$

80010001200

$\lambda[\mathrm{nm}]$

Fig. 5. Theoretical modulation matrix of ProMag. The crosses indicate the measured modulation amplitudes at 587.6 and $1083.0 \mathrm{~nm}$. The first column of the matrix is identically equal to 1 and is not shown.

proving the modulation efficiency of existing polarimetric instruments, as well as by designing new modulators with near optimal and balanced efficiency over very large spectral ranges. Finally we have demonstrated the applicability of this new paradigm by showing the measured performance of a prototype modulator, which was designed and optimized through the method presented in this paper.

\section{Acknowledgments}

We thank D. Elmore for helpful discussions during the early stages of this work, S. Sewell for helpful comments on the manuscript and Frans Snik for pointing us to the Yunnan polarimeter paper. The National Center for Atmospheric Research is sponsored by the National Science Foundation.

\section{References}

1. G. E. Hale, "On the probable existence of a magnetic field in sunspots," Astrophys. J. 28, 315-343 (1908).

2. J. O. Stenflo, "The Hanle effect and the diagnostics of turbulent magnetic fields in the solar atmosphere," Solar Phys. 80, 209-226 (1982).

3. J. Trujillo Bueno, N. Shchukina, and A. Asensio Ramos, "A substantial amount of hidden magnetic energy in the quiet Sun," Nature 430, 326-329 (2004). 
4. M. Faurobert-Scholl, "Investigation of microturbulent magnetic fields in the solar photosphere by their Hanle effect in the Sr I 4607 Åline," Astron. Astrophys. 268, 765-774 (1993).

5. J. O. Stenflo, and C. U. Keller, "The second solar spectrum. A new window for diagnostics of the Sun," Astron. Astrophys. 321, 927-934 (1997).

6. A. López Ariste, S. Tomczyk, and R. Casini, "Hyperfine structure as a diagnostic of solar magnetic fields," Astrophys. J. 580, 519-527 (2002).

7. A. López Ariste, S. Tomczyk, and R. Casini, "Quiet sun magnetic field diagnostics with a Mn line," Astron. Astrophys. 454, 663-668 (2006).

8. A. Asensio Ramos, M. Martínez González, A. López Ariste, J. Trujillo Bueno, and M. Collados, "A near-infrared line of Mn I as a diagnostic tool of the average magnetic energy in the solar photosphere," Astrophys. J. 659, 829-847 (2007).

9. J. Štěpán, P. Heinzel, and S. Sahal-Bréchot, "Hydrogen $\mathrm{H} \alpha$ line polarization in solar flares. Theoretical investigation of atomic polarization by proton beams considering self-consistent NLTE polarized radiative transfer," Astron. Astrophys. 465, 621-631 (2007).

10. J. O. Stenflo, "Magnetic-field structure of the photospheric network," Solar Phys. 32, 41-63 (1973).

11. J. O. Stenflo, S. K. Solanki, and J. W. Harvey, "Diagnostics of solar magnetic fluxtubes with the infrared line Fe I $\lambda 15648.54 \AA$," Astron. Astrophys. 173, 167-179 (1987).

12. I. Rüedi, S. K. Solanki, W. Livingston and J. W. Harvey, "Interesting lines in the infrared solar spectrum. III. A polarimetric survey between 1.05 and $2.50 \mu \mathrm{m}$," Astron. Astrophys. 113, 91-106 (1995).

13. H. Socas-Navarro, D. F. Elmore, A. Pietarila, T. Darnell, B. W. Lites, S. Tomczyk, and S. Hegwer, "SPINOR: Visible and infrared spectro-polarimetry at the National Solar Observatory," Solar Phys., 235, 55-73 (2006).

14. P. Goode, C. J. Denker, L. I. Didkovsky, J. R. Kuhn and H. Wang, "1.6 m Solar Telescope in Big Bear - The NST," J. Korean Ast. Soc. 36, 125-133 (2003).

15. S. L. Keil, T. Rimmele, C. U. Keller, F. Hill, R. R. Radick, J. M. Oschmann, M. Warner, N. E. Dalrymple, J. Briggs, S. Hegwer and D. Ren, "Design and development of the Advanced Technology Solar Telescope (ATST)," Proc. SPIE 4853, 240-251 (2003).

16. M. Collados, "European Solar Telescope (EST): project status," Proc. SPIE 7012, 70120J (2008).

17. A. V. Samoylov, V. S. Samoylov, A. P. Vidmachenko, and A. V. Perekhod, "Achromatic and super-achromatic zero-order waveplates," JQSRT 88319325 (2004).

18. J. Ma, J. S. Wang, C. Denker, and H. M. Wang, "Optical design of multilayer achromatic waveplate by simulated annealing algorithm," Chinese J. Astron. Astrophys. 8, 349-361 (2008).

19. M. Collados, "High resolution spectropolarimetry and magnetography," in 3rd Advances in Solar Physics Euroconference,B. Schmieder, A. Hoffman, J. Staude, eds. (ASP Conf. Ser. 184), pp. 3-22 (1999).

20. J. C. del Toro Iniesta and M. Collados, "Optimum modulation and demodulation matrices for 
solar polarimetry," Appl. Opt. 39, 1637-1642 (2000).

21. S. Tomczyk, G. L. Card, T. Darnell, D. F. Elmore, R. Lull, P. G. Nelson, K. V. Streander, J. Burkepile, R. Casini and P. G. Judge, "An instrument to measure coronal emission line polarization," Solar Phys. 247, 411-428 (2008).

22. K. Sankarasubramanian, B. Lites, C. Gullixson, D. F. Elmore, S. Hegwer, K. V. Streander, T. Rimmele, S. Fletcher, S. Gregory and M. Sigwarth, "The Diffraction Limited SpectroPolarimeter," in Solar Polarization 4, R. Casini and B. Lites, eds. (ASP Conf. Ser. 358), pp. 201-204 (2006).

23. Y. Hanaoka, "Ferroelectric liquid crystal polarimeter for high-cadence H $\alpha$ imaging polarimetry," Solar Phys. 222, 265-278 (2004).

24. D. Elmore, National Solar Observatory, private communication (2008).

25. A. M. Gandorfer, "Ferroelectric retarders as an alternative to piezoelastic modulators for use in solar Stokes vector polarimetry," Opt. Eng. 38, 1402-1408 (1999).

26. D. Gisler, A. Feller and A. Gandorfer, "Achromatic liquid crystal polarization modulator," Proc. SPIE 4843, 45-54 (2003).

27. C. Xu, Z. Qu, X. Zhang, C. Jin and X. Yan, "Polarimeter with two ferroelectric liquid-crystal modulators attached to the Yunnan solar tower," Appl. Opt. 45, 8428-8433 (2006).

28. M. D. McKay, R. J. Beckman and W. J. Conover, "A Comparison of Three Methods for Selecting Values of Input Variables in the Analysis of Output from a Computer Code," Technometrics 21, 239-245 (1979).

29. B. W. Lites, "Rotating waveplates as polarization modulators for Stokes polarimetry of the sun - Evaluation of seeing-induced crosstalk errors," Appl. Opt. 26, 3838-3845 (1987).

30. D. F. Elmore, R. Casini, G. L. Card, M. Davis, A. Lecinski, R. Lull, P. G. Nelson and S. Tomczyk, "A new spectro-polarimeter for solar prominence and filament magnetic field measurements," Proc. SPIE 7014, 701416 (2008). 